\title{
THE CONCEPT OF NORMALITY FOR FUZZY RANDOM VARIABLES ${ }^{1}$
}

\author{
By Madan L. Puri and Dan A. Ralescu \\ Indiana University and University of Cincinnati
}

\begin{abstract}
In this paper we define the concept of a normal fuzzy random variable and we prove the following representation theorem: Every normal fuzzy random variable equals the sum of its expected value and a mean zero random vector.
\end{abstract}

1. Introduction. The concept of a fuzzy random variable [cf., Puri and Ralescu (1984)] was defined as a tool for representing relationships between the outcomes of a random experiment and inexact data. By inexactness here we mean nonstatistical inexactness that is due to subjectivity and imprecision of human knowledge rather than to the occurrence of random events. Fuzzy random variables generalize the concept of a random variable as well as that of a random set [Matheron (1975)].

To make this theory applicable to statistical analysis of inexact data, we consider in this paper the concept of normality for fuzzy random variables. [For the concept of normality of random sets, the reader is referred to Lyashenko (1980). For a characterization of $p$-stable random sets, the reader is referred to Giné and Hahn (1985).]

After some preliminaries on fuzzy random variables given in Section 2, we describe in Section 3 an embedding theorem for the space of fuzzy sets with compact convex levels that satisfy a certain Lipschitz condition. In Section 4, we define the concept of a normal fuzzy random variable in $\mathbb{R}^{p}$ and represent such a variable as the sum of a constant fuzzy set and a random vector in $\mathbb{R}^{p}$. This result extends the corresponding result for random sets [Lyashenko (1980)] and, analogous to his result, it shows that a normal fuzzy random variable is "degenerate" in the sense that it is a random translate of a fixed fuzzy set. Our result is also analogous to the one given in Giné and Hahn (1985) which deals with random sets in Banach space.

2. Fuzzy random variables. Let $\mathscr{K}\left(\mathbb{R}^{p}\right)$ denote the nonempty compact subsets of $\mathbb{R}^{p}$, and let $\mathscr{K}_{c}\left(\mathbb{R}^{p}\right)$ denote the nonempty compact convex subsets of $\mathbb{R}^{p}$. The Hausdorff distance

$$
d(A, B)=\max \left\{\sup _{a \in A} \inf _{b \in B}\|a-b\|, \sup _{b \in B} \inf _{a \in A}\|a-b\|\right\}
$$

gives $\mathscr{K}\left(\mathbb{R}^{p}\right)$ a structure of a metric space. Moreover, this space is complete and separable, and $\mathscr{K}_{c}\left(\mathbb{R}^{p}\right)$ is a closed subspace [Debreu, (1967)].

Received May 1984; revised February 1985.

${ }^{1}$ Research supported by a National Science Foundation Grant.

AMS 1980 subject classifications. Prìmary 60D05; secondary $03 \mathrm{E} 72$.

Key words and phrases. Fuzzy set, fuzzy random variable, embedding, Gaussian element. 
Denote $\|A\|=d(A,\{0\})$ for $A \in \mathscr{K}\left(\mathbb{R}^{p}\right)$. A linear structure is defined in $\mathscr{K}\left(\mathbb{R}^{p}\right)$ by the operations

$$
A+B=\{a+b \mid a \in A, b \in B\}, \quad \lambda A=\{\lambda a \mid a \in A\},
$$

where $A, B \in \mathscr{K}\left(\mathbb{R}^{p}\right)$ and $\lambda \in \mathbb{R}$. Note that $\mathscr{K}\left(\mathbb{R}^{p}\right)$ is not a vector space.

Let $(\Omega, \mathscr{A}, P)$ be a probability space. A random set is a Borel measurable function $f: \Omega \rightarrow \mathscr{K}\left(\mathbb{R}^{p}\right)$. The expected value Ef [Aumann (1965)] is defined by

$$
E f=\left\{E \phi \mid \phi \in L^{1}(\Omega, \mathscr{A}, P), \phi(\omega) \in f(\omega) \text { a.e. }\right\},
$$

where $\phi: \Omega \rightarrow \mathbb{R}^{p}$ is a selection of $f$.

Note that $E\|f\|<\infty$ implies that $E f \in \mathscr{K}\left(\mathbb{R}^{p}\right)$.

A fuzzy set in $\mathbb{R}^{p}$ is a function $u: \mathbb{R}^{p} \rightarrow[0,1]$ [Zadeh (1965)]. Denote by $L_{\alpha}(u)=\left\{x \in \mathbb{R}^{p} \mid u(x) \geq \alpha\right\}$ for $0 \leq \alpha \leq 1$, the level sets of $u$. Further, denote by supp $u$, the support of $u$, i.e., the closure of the set $\left\{x \in \mathbb{R}^{p} \mid u(x) \neq 0\right\}$.

As an extension of $\mathscr{K}\left(\mathbb{R}^{p}\right)$ we define the space $\mathscr{F}\left(\mathbb{R}^{p}\right)$ of fuzzy sets $u: \mathbb{R}^{p} \rightarrow[0,1]$ with the properties:

(a) $u$ is upper semicontinuous;

(b) $\operatorname{supp} u$ is compact;

(c) $\left\{x \in \mathbb{R}^{p} \mid u(x)=1\right\} \neq \phi$.

The linear structure in $\mathscr{F}\left(\mathbb{R}^{p}\right)$ is defined by the operations $(u+v)(x)=$ $\sup _{y+z=x} \min [u(y), v(z)]$,

$$
(\lambda u)(x)= \begin{cases}u\left(\lambda^{-1} x\right), & \text { if } \lambda \neq 0, \\ \chi_{\{0\}}(x), & \text { if } \lambda=0,\end{cases}
$$

where $u, v \in \mathscr{F}\left(\mathbb{R}^{p}\right), \lambda \in \mathbb{R}$, and $\chi_{A}$ denotes the characteristic function of $A \subset \mathbb{R}^{p}$.

Note that $L_{\alpha}(u+v)=L_{\alpha}(u)+L_{\alpha}(v)$ and $L_{\alpha}(\lambda u)=\lambda L_{\alpha}(u)$ for every $0 \leq \alpha$ $\leq 1$.

We extend the Hausdorff metric by defining $d_{\infty}(u, v)=\sup _{\alpha>0} d\left(L_{\alpha} u, L_{\alpha} v\right)$; $u, v \in \mathscr{F}\left(\mathbb{R}^{p}\right)$ (see Puri and Ralescu, 1981 and 1983).

It is possible to show that $\left(\mathscr{F}\left(\mathbb{R}^{p}\right), d_{\infty}\right)$ is complete [Puri and Ralescu (1984)].

Denote by $\mathscr{F}_{c}\left(\mathbb{R}^{p}\right)$ the space of fuzzy sets $u \in \mathscr{F}\left(\mathbb{R}^{p}\right)$ such that $L_{\alpha}(u)$ is convex for each $\alpha \geq 0$.

A fuzzy random variable is a Borel measurable function $X: \Omega \rightarrow\left(\mathscr{F}\left(\mathbb{R}^{p}\right), d_{\infty}\right)$. If $E\|\operatorname{supp} X\|<\infty$, then the expected value $E X$ is the unique fuzzy set satisfying the property

$$
L_{\alpha}(E X)=E\left(L_{\alpha} X\right), \quad 0<\alpha \leq 1
$$

[Puri and Ralescu (1984), Theorem 3.1]. It also follows that $E\|\operatorname{supp} X\|<\infty$ implies $E X \in \mathscr{F}\left(\mathbb{R}^{p}\right)$.

A strong law of large numbers and a central limit theorem for fuzzy random variables were derived in Klement, Puri, and Ralescu (1985a, b). A law of large numbers was also given by Kruse (1982) using a different concept of expected value. 
3. The embedding theorem. Let $S^{p-1}=\left\{x \in \mathbb{R}^{p} \mid\|x\|=1\right\}$ denote the unit sphere in $\mathbb{R}^{p}$. It is well known that $\mathscr{K}_{c}\left(\mathbb{R}^{p}\right)$ can be embedded isometrically into $C\left(S^{p-1}\right)$ (the Banach space of continuous functions on $S^{p-1}$ ). This result goes back to Minkowski [see, e.g., Artstein and Vitale (1975)].

Such an embedding is realized via the support function of a compact convex set. More precisely, if $K \in \mathscr{K}_{c}\left(\mathbb{R}^{p}\right)$, its support function is defined by

$$
s_{K}(x)=\sup _{a \in K}\langle x, a\rangle, \quad x \in S^{p-1},
$$

where $\langle\cdot, \cdot\rangle$ is the inner product in $\mathbb{R}^{p}$.

In the case of the space $\mathscr{F}_{c}\left(\mathbb{R}^{p}\right)$ of fuzzy sets, we seek a similar embedding. However, more restrictions need to be imposed to do this.

Consider the space $\mathscr{F}_{C L}\left(\mathbb{R}^{p}\right)$ of fuzzy sets $u \in \mathscr{F}_{c}\left(\mathbb{R}^{p}\right)$ such that the map $\alpha \rightarrow L_{\alpha}(u)$ is Lipschitz with respect to the Hausdorff distance. More precisely, $u \in \mathscr{F}_{C L}\left(\mathbb{R}^{p}\right)$ if there exists a constant $M>0$, such that $d\left(L_{\alpha} u, L_{\beta} u\right) \leq M|\alpha-\beta|$ for every $\alpha, \beta \in(0,1]$.

The following theorem gives the desired embedding, which will be used in the next section to define and characterize fuzzy random variables.

THEOREM 3.1. There exists a function

$$
j: \mathscr{F}_{C L}\left(\mathbb{R}^{p}\right) \rightarrow C\left([0,1] \times S^{p-1}\right)
$$

such that:

(i) $j$ is an isometry (i.e., $\left.\|j(u)-j(v)\|_{\infty}=d_{\infty}(u, v)\right)$;

(ii) $j(u+v)=j(u)+j(v)$;

(iii) $j(\lambda u)=\lambda j(u), \lambda \geq 0$.

Proof. Define $j(u)=s_{u}$, where

$$
s_{u}(\alpha, x)= \begin{cases}s_{L_{\alpha} u}(x), & \text { if } \alpha>0, \\ s_{\text {supp } u}(x), & \text { if } \alpha=0 .\end{cases}
$$

The function $s_{u}$ is Lipschitz on $[0,1] \times S^{p-1}$ :

$$
\begin{aligned}
\left|s_{u}(\alpha, x)-s_{u}(\beta, y)\right| & \leq\left|s_{L_{\alpha} u}(x)-s_{L_{\beta} u}(x)\right|+\left|s_{L_{\beta} u}(x)-s_{L_{\beta} u}(y)\right| \\
& \leq\left\|s_{L_{\alpha} u}-s_{L_{\beta} u}\right\|_{\infty}+\left\|L_{\beta} u\right\|\|x-y\| \\
& \leq\|u\|_{L}(|\alpha-\beta|+\|x-y\|),
\end{aligned}
$$

where

$$
\|u\|_{L}=\sup _{\alpha \neq \beta} \frac{d\left(L_{\alpha} u, L_{\beta} u\right)}{|\alpha-\beta|}+\sup _{\alpha>0}\left\|L_{\alpha} u\right\|
$$

and we have used the fact that $K \in \mathscr{K}_{c}\left(\mathbb{R}^{p}\right) \rightarrow s_{K}$ is an isometry, as well as the fact that $s_{K}$ satisfies a Lipschitz condition with constant $\|K\|$. 
Since any two norms in $\mathbb{R}^{p+1}$ are equivalent, inequality (3.1) becomes

$$
\left|s_{u}(\alpha, x)-s_{u}(\beta, y)\right| \leq q\|u\|_{L}\left(|\alpha-\beta|^{2}+\|x-y\|^{2}\right)^{1 / 2},
$$

where $q$ is a constant.

Thus $s_{u}=j(u) \in C\left([0,1] \times S^{p-1}\right)$.

It is now easy to show that $j$ is an isometry, i.e.,

$$
\|j(u)-j(v)\|_{\infty}=\sup _{\alpha, x}\left|s_{u}(\alpha, x)-s_{v}(\alpha, x)\right|=d_{\infty}(u, v),
$$

as well as properties (ii) and (iii), and the proof is completed.

Note. $s_{u}$ can also be defined on $[0,1] \times \mathbb{R}^{p}$ by the same formula as above, and it satisfies the following properties

(a) $s_{u}(\alpha, \cdot)$ is subadditive, i.e., $s_{u}(\alpha, x+y) \leq s_{u}(\alpha, x)+s_{u}(\alpha, y)$ for $x, y \in \mathbb{R}^{p}$.

(b) $s_{u}(\alpha, \cdot)$ is positively homogeneous, i.e., $s_{u}(\alpha, \lambda x)=\lambda s_{u}(\alpha, x)$ for $x \in \mathbb{R}^{p}$, $\lambda \geq 0$.

(c) $s_{u}(\cdot, x)$ is decreasing.

(d) $s_{u}(\cdot, x)$ is left-continuous.

Properties (a), (b), and (c) will be used in the proof of our representation theorem in Section 4.

From Theorem 3.1, it follows that every fuzzy random variable $X: \Omega \rightarrow$ $\mathscr{F}_{C L}\left(\mathbb{R}^{p}\right)$ can be thought of as a random element of $C\left([0,1] \times S^{p-1}\right)$ by considering $j(X)=s_{X}: \Omega \rightarrow C\left([0,1] \times S^{p-1}\right)$ where $s_{X}(\omega)=s_{X(\omega)}$.

The following result will also be useful.

Lemma 3.1. If $X: \Omega \rightarrow \mathscr{F}_{C L}\left(\mathbb{R}^{p}\right)$ satisfies $E\|\operatorname{supp} X\|<\infty$, then $E\left[s_{X}(\alpha, x)\right]$ $=s_{E X}(\alpha, x)$, for $\alpha \in[0,1], x \in \mathbb{R}^{p}$.

Proof. Let $\alpha_{0} \in[0,1]$ be fixed and consider the random set $f=L_{\alpha_{0}}(X)$. We first show that $E\left[s_{f}(x)\right]=s_{E f}(x)$ for each $x \in S^{p-1}$. Indeed, it is easy to see that this result holds if $f=\sum_{i=1}^{n} \chi_{A_{i}} K_{i}$ where $A_{i} \in \mathscr{A}, K_{i} \in \mathscr{K}_{c}\left(\mathbb{R}^{p}\right)$, and $\chi_{A_{i}}$ denotes the characteristic function of $A_{i}$ (i.e., if $f$ is a simple function). The desired formula follows by using the expected value of Debreu (1967), the fact that $K \in \mathscr{K}_{c}\left(\mathbb{R}^{p}\right) \rightarrow s_{K}$ is an isometry, and the Lebesgue dominated convergence theorem. Thus

$$
E\left[s_{L_{\alpha_{0}} X}(x)\right]=s_{E\left(L_{\alpha_{0} X} X\right.}(x)=s_{L_{\alpha_{0}}(E X)}(x)=s_{E X}\left(\alpha_{0}, x\right),
$$

implying $E\left[s_{X}\left(\alpha_{0}, x\right)\right]=s_{E X}\left(\alpha_{0}, x\right)$, the desired result. We have used the property $E\left(L_{\alpha} X\right)=L_{\alpha}(E X)$ of the expected value $E X$.

4. Normal fuzzy random variables. In this section we define the concept of a normal fuzzy random variable (by using the embedding of Section 3), and we prove that such a variable can be uniquely represented as the sum of a constant fuzzy set and a normal random vector in $\mathbb{R}^{p}$. 
Before doing this, we shall need the following preliminaries: Let $C(M)$ denote the space of continuous functions on the compact set $M$.

A random element $f: \Omega \rightarrow C(M)$ is Gaussian if the vector $\left(f\left(t_{1}\right), \ldots, f\left(t_{n}\right)\right)$ is normal for any $t_{1}, \ldots, t_{n} \in M$.

Definition 4.1. A fuzzy random variable $X: \Omega \rightarrow \mathscr{F}_{C L}\left(\mathbb{R}^{p}\right)$ is normal if $s_{X}$ is a Gaussian random element of $C\left([0,1] \times S^{p-1}\right)$.

This definition and the comment above imply that $\left(s_{X}\left(\alpha_{1}, x_{1}\right), \ldots, s_{X}\left(\alpha_{n}, x_{n}\right)\right)$ is normal for every $\alpha_{1}, \ldots, \alpha_{n} \in[0,1]$ and $x_{1}, \ldots, x_{n} \in S^{p-1}$. Since $s_{X}(\alpha, x)$ is positively homogeneous in $x$, it follows that $\left(s_{X}\left(\alpha_{1}, x_{1}\right), \ldots, s_{X}\left(\alpha_{n}, x_{n}\right)\right)$ is normal for every $\alpha_{1}, \ldots, \alpha_{n} \in[0,1]$ and $x_{1}, \ldots, x_{n} \in \mathbb{R}^{p}$. It also follows from Definition 4.1 that if $X$ and $Y$ are independent normal fuzzy random variables, then $X+Y$ is normal. Also $\lambda X$ is normal whenever $X$ is normal and $\lambda \in \mathbb{R}$.

REMARK. This definition of normality for fuzzy random variables is an extension of the concept of normality of random vectors in $\mathbb{R}^{p}$ as well as the concept of a normal random set [Lyashenko (1980)]. Definition 4.1 is a natural one, in view of the embedding Theorem 3.1, since in this way, normality is defined via the random element $s_{X}$ that takes values in the familiar Banach space $C\left([0,1] \times S^{p-1}\right)$.

We now prove the characterization theorem for normal fuzzy random variables.

THEOREM 4.1. Let $X: \Omega \rightarrow \mathscr{F}_{C L}\left(\mathbb{R}^{p}\right)$ be a fuzzy random variable with $E\|\operatorname{supp} X\|<\infty$. The following statements are equivalent.

(1) $X$ is normal.

(2) $X=E X+\{\xi\}$ where $\xi$ is a normal random vector with mean zero.

Proof. (2) $\Rightarrow(1)$. It is easily seen that if $\alpha_{1}, \ldots, \alpha_{n} \in[0,1]$ and $x_{1}, \ldots, x_{n} \in$ $S^{p-1}$, then $\left(s_{X}\left(\alpha_{1}, x_{1}\right), \ldots, s_{X}\left(\alpha_{n}, x_{n}\right)\right)=M \xi+b$ where $M$ is a constant matrix and $b$ is a constant vector. Since $M \xi+b$ is normal, it follows that $s_{X}$ is Gaussian in $C\left([0,1] \times S^{p-1}\right)$.

$(1) \Rightarrow(2)$. Let $s_{X}$ be Gaussian. Fix $\omega \in \Omega$ and $\alpha \in[0,1]$. Define $\psi(\omega, \alpha)=$ $s_{X(\omega)}(\alpha, \cdot)-s_{E X}(\alpha, \cdot)$. Thus $\psi(\omega, \alpha): \mathbb{R}^{p} \rightarrow \mathbb{R}$.

Claim 1. $\psi(\omega, \alpha)$ is linear.

Let $\lambda, \mu \geq 0$, and $x_{1}, x_{2} \in \mathbb{R}^{p}$ be fixed. Then

$$
\lambda s_{X}\left(\alpha, x_{1}\right)+\mu s_{X}\left(\alpha, x_{2}\right)-s_{X}\left(\alpha, \lambda x_{1}+\mu x_{2}\right) \geq 0,
$$

since $s_{X}(\alpha, x)$ is positively homogeneous in $x$ as well as subadditive [properties (a) and (b) of Section 3].

By hypothesis, the vector $\left(s_{X}\left(\alpha, x_{1}\right), s_{X}\left(\alpha, x_{2}\right), s_{X}\left(\alpha, \lambda x_{1}+\mu x_{2}\right)\right)$ is normal, which implies that $\lambda s_{X}\left(\alpha, x_{1}\right)+\mu s_{X}\left(\alpha, x_{2}\right)-s_{X}\left(\alpha, \lambda x_{1}+\mu x_{2}\right)$ is a normal random variable. From (4.1), it follows that this random variable is degenerate, i.e., 
$\lambda s_{X}\left(\alpha, x_{1}\right)+\mu s_{X}\left(\alpha, x_{2}\right)-s_{X}\left(\alpha, \lambda x_{1}+\mu x_{2}\right)=E\left[\lambda s_{X}\left(\alpha, x_{1}\right)+\mu s_{X}\left(\alpha, x_{2}\right)-\right.$ $\left.s_{X}\left(\alpha, \lambda x_{1}+\mu x_{2}\right)\right]=\lambda s_{E X}\left(\alpha, x_{1}\right)+\mu s_{E X}\left(\alpha, x_{2}\right)-s_{E X}\left(\alpha, \lambda x_{1}+\mu x_{2}\right)$. The last equality follows from Lemma 3.1. In terms of our function $\psi(\omega, \alpha)$, this implies that $\psi(\omega ; \alpha)\left(\lambda x_{1}+\mu x_{2}\right)=\lambda \psi(\omega, \alpha)\left(x_{1}\right)+\mu \psi(\omega, \alpha)\left(x_{2}\right)$. Since $\psi(\omega, \alpha)(0)=0$, this proves the linearity of $\psi(\omega, \alpha)$.

It is well known that such a linear map from $\mathbb{R}^{p}$ into $\mathbb{R}$ can be represented as $\psi(\omega, \alpha)(x)=\left\langle x, \xi_{\alpha}(\omega)\right\rangle, x \in \mathbb{R}^{p}$ for some $\xi_{\alpha}(\omega) \in \mathbb{R}^{p}$.

Claim 2. $\xi_{\alpha}(\omega)$ does not depend on $\alpha \in[0,1]$.

Let $\alpha, \beta \in[0,1], \alpha \leq \beta$ fixed. From property (c) of Section 3, it follows that $s_{X}(\alpha, x) \geq s_{X}(\beta, x)$ for every $x \in \mathbb{R}^{p}$. But (using the same argument as before) $s_{X}(\alpha, x)-s_{X}(\beta, x)$ is a normal random variable, which implies that $s_{X}(\alpha, x)-s_{X}(\beta, x)=E\left[s_{X}(\alpha, x)-s_{X}(\beta, x)\right]=s_{E X}(\alpha, x)-s_{E X}(\beta, x)$. Thus $\psi(\omega, \alpha)(x)=\psi(\omega, \beta)(x)$ for every $x \in \mathbb{R}^{p}$. This shows that $\xi_{\alpha}(\omega)=\xi(\omega)$ is independent of $\alpha$.

Thus we have the representation $\psi(\omega, \alpha)(x)=\langle x, \xi(\omega)\rangle$. Since $\langle x, \xi\rangle$ is measurable for each $x \in \mathbb{R}^{p}$, it follows that $\xi: \Omega \rightarrow \mathbb{R}^{p}$ is measurable. Now, from the representation $\langle x, \xi\rangle=s_{X}(\alpha, x)-s_{E X}(\alpha, x)$, it follows that $\langle x, \xi\rangle$ is a normal mean zero random variable for each $x \in \mathbb{R}^{p}$. Thus $\xi$ is a normal mean zero random vector.

To conclude the proof of the theorem, we write $s_{X}(\alpha, x)=s_{E X}(\alpha, x)+\langle x, \xi\rangle$ $=s_{E X}(\alpha, x)+s_{\{\xi\}}(\alpha, x)=s_{E X+\{\xi\}}(\alpha, x)$, implying that $X=E X+\{\xi\}$.

\section{REFERENCES}

[1] ARtstein, Z. and Vitale, R. A. (1975). A strong law of large numbers for random compact sets. Ann. Probab. 3 879-882.

[2] Aumann, R. J. (1965). Integrals of set-valued functions. J. Math. Anal. Appl. 12 1-12.

[3] Debreu, G. (1967). Integration of correspondences. Proc. Fifth Berkeley Symp. Math. Statist. Probability II 351-372. Univ. Calif. Press.

[4] GiNE E. and HAHN, M. G. (1985). Characterization and domains of attraction of $p$-stable random compact sets. Ann. Probab. 13 447-468.

[5] Klement, E. P. Puri, M. L. and Ralescu, D. A. (1985a). Law of large numbers for fuzzy random variables. Technical report, Department of Mathematics, Indiana University.

[6] Klement, E. P., Puri, M. L. and Ralescu, D.A. (1985b). Central limit theorems for fuzzy random variables. Technical report, Department of Mathematics, Indiana University.

[7] KRUSE, R. (1982). The strong law of large numbers for fuzzy random variables. Inform. Sci. 28 233-241.

[8] Lyashenko, N. N. (1980). Statistics of random compacts in euclidean space. Zap. Naucn. Sem. Leningrad. Otdel. Mat. Inst. Steklov. 98 115-139. (English translation in J. Soviet Math. 21 (1983) 76-92.

[9] Matheron, G. (1975). Random Sets and Integral Geometry. Wiley, New York.

[10] Puri, M. L. and Ralescu, D. A. (1981). Différentielle d'une fonction floue. C. R. Acad. Sc. Paris, t. 293, Série I, 237-239.

[11] Puri, M. L. and Ralescu, D. A. (1982). Integration on fuzzy sets. Adv. in Appl. Math. 3 430-434.

[12] Puri, M. L. and Ralescu, D. A. (1983). Differentials of fuzzy functions. J. Math. Anal. Appl. 91 552-558. 
[13] Puri, M. L. and Ralescu, D. A. (1984). Fuzzy random variables. To appear in J. Math. Anal. Appl. (January 1986 issue).

[14] WeIL, W. (1982). An application of the central limit theorem for Banach space-valued random variables to the theory of random sets. Z. Wahrsch. verw. Gebiete 60 203-208.

[15] ZADEH, L. A. (1965). Fuzzy sets. Inform. and Control 8 338-353.

DePaRTMENT OF Mathematics

INDIANA UNIVERSITY

BLOOMINGTON, INDIANA 47405
DEPARTMENT OF MATHEMATICS UNIVERSITY OF CINCINNATI

CINCINNATI, OHIO 45221 